\title{
VELOCITY AND GRAVITATIONAL EFFECTS ON GPS SATELLITES: AN OUTLINE OF EARLY PREDICTION AND DETECTION OF STRONG EARTHQUAKES
}

\author{
EFECTOS DE VELOCIDAD Y DE GRAVITACIÓN EN GPS SATELITALES: UN \\ ESQUEMA PARA LA PREDICCIÓN Y DETECCIÓN TEMPRANA DE FUERTES \\ TERREMOTOS
}

\author{
H. Torres-Silva ${ }^{1} \quad$ A. Souza de Assis ${ }^{2}$ \\ Recibido 11 de marzo de 2010, aceptado 17 de noviembre de 2010 \\ Received: March 11, 2010 Accepted: November 17, 2010 \\ RESUMEN
}

\begin{abstract}
Hoy en día, los sistemas de navegación global por satélite, GPS utilizados como sistemas de posicionamiento global, se basan en un modelo gravitacional y por lo tanto solo son operativos cuando varios efectos relativistas son tenidos en cuenta. Los efectos relativistas más importantes (hasta el orden $1 / \mathrm{c}^{2}$ ) son: el desplazamiento Doppler al rojo de segundo orden, debido al movimiento del satélite (la relatividad especial) y el efecto gravitacional de Einstein corrimiento al azul de la frecuencia de reloj del satélite (principio de equivalencia de la relatividad general). Ambos efectos pueden ser tratados en un nivel básico, apelando a la relatividad del día a día. Este artículo examina los efectos significativos que deben tenerse en cuenta en la operación de sistemas de GPS sin tener que recurrir a las teorías de la relatividad especial y general, produciendo los mismos resultados para estos sistemas, donde uno de los efectos puede ser tratado con el enfoque temporal de la contracción que aquí se propone y el otro usando la teoría de Newton como una aproximación de la Relatividad General. Este enfoque nos permite proponer un esquema de la predicción y detección temprana de los fenómenos de terremoto de gran intensidad.
\end{abstract}

Palabras clave: Relatividad, GPS, gravitación, terremotos, predictor.

\begin{abstract}
Today, the global navigation satellite systems, GPS used as global positioning systems, are based on a gravitational model and hence they are only operative when several relativistic effects are taken into account. The most important relativistic effects (to order $1 / \mathrm{c}^{2}$ ) are: the Doppler red shift of second order, due to the motion of the satellite (special relativity) and the Einstein gravitational blue shift effect of the satellite clock frequency (equivalence principle of general relativity). Both of these effects can be treated at a basic level, making for an appealing application of relativity to every life. This paper examines the significant effects that must be taken into account in the design and operation of systems GPS without resorting to the theory of special and general relativity, yielding the same results for these systems, where one of the effects can be treated with the time contraction approach proposed here and the other using the Newton's theory as an approximation of the General Relativity. This approach allow us to propose an outline of early prediction and detection on strong earthquake phenomena.
\end{abstract}

Keywords: Relativity, GPS, gravitation, earthquake, predictor.

\section{INTRODUCTION}

The global positioning system (GPS) provides a superb opportunity to introduce relativity concepts to undergraduate students, including non-physics majors. Familiarity with the numerous applications of GPS motivates students to understand relativity. A few fundamental principles need to be introduced, including the postulates of special relativity and the universality of free fall. A series of effects can lead to the breakdown of simultaneity, the Sagnac effect, the first-order Doppler effect, gravitational frequency shifts, and time dilation.

Important relativistic effects on GPS satellite clocks include gravitational frequency shifts and time dilation. These effects are so large that if not accounted for, the system

\footnotetext{
1 Instituto de Alta Investigación. Universidad de Tarapacá. Arica, Chile. E-mail: htorres@uta.cl

2 Departamento de Matemática Aplicada. Universidade Federal do Rio de Janeiro. Rio de Janeiro, RJ, Brasil.
} 
would not be effective for navigation. Reference clocks on earth's geoid are similarly influenced by time dilation (due to earth's rotation) and gravitational frequency shifts, relative to clocks at infinity.

The frequency differences between clocks in orbit, and reference clocks on earth's surface, are very important in the GPS. Constancy of the speed of light is essential for navigation using GPS. This principle also leads directly to the relativity of simultaneity and to the Sagnac effect, that must be accounted for when synchronizing clocks in the neighborhood of earth or comparing clocks that are thousands of km apart on earth's surface but that have one or more GPS satellites in view at the same time. The relativity of simultaneity, the constancy of c, and the firstorder Doppler effect are intimately related.

This article presents a different approach to explain the essential role of special and general relativity in the GPS. This is made through the time contraction and the Newton Theory considered as an approximation of the Einstein's General Relativity.

\section{TIME CONTRACTIONS AND VELOCITY EFFECT}

We present here a simple conjecture to show how time and information behaves in motion objects (particles or structures). In order to do that we considerer (using a restricted relativity principle) that information can propagate at the maximum light speed velocity (c).

We construct now a information/ communication diagram for interacting systems $\mathrm{A}$ and $\mathrm{B}$. The system $\mathrm{A}$ is at rest and the system $\mathrm{B}$ movies at constant velocity v. Considerer also that information is realized by $\mathrm{A}$ to $\mathrm{B}$ which propagates at light velocity c. Also considerer that the system A possess two clock synchronized at time $\mathrm{t}=0$.

The position B' reads for the position of object B when information realized from $\mathrm{A}$ reaches its $\mathrm{t}=0$ position.

The angle $\alpha$ is the triangulation angle, it means the angle that creates the simultaneity of signal communication or, for binary information system (two elementary particles synchronizing information via entangled photons). When both systems receive the information of each other we have a awareness time. The time t' marks this awareness instant (in this case both send information when $t^{\prime} \rightleftarrows A B^{\prime}$, $\left.c \rightarrow B^{\prime}, \ldots \alpha=\pi / 2\right)$.

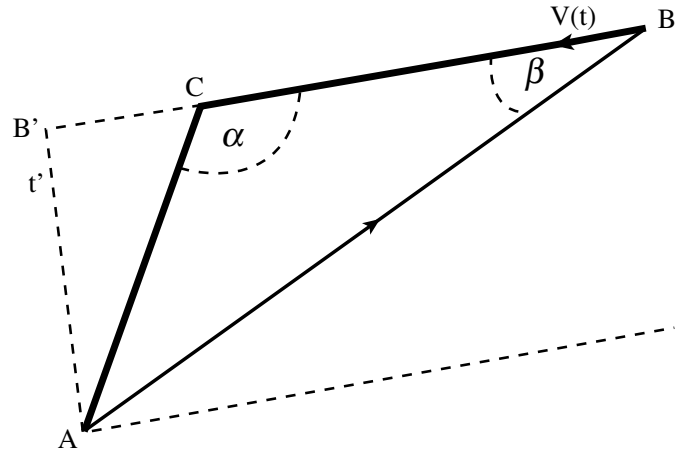

Figure 1. The cosines law to study the relativity of simultaneity.

Using, therefore, the cosines law (see the diagram 1), we can write

$$
c^{2} t^{2}=v^{2} t^{2}+c^{2} t^{\prime 2}-2 v c t t^{\prime} \cos \alpha
$$

Therefore, for $\Delta t^{\prime}$ one gets

$$
\Delta t^{\prime}=\frac{v}{c} \Delta t \cos \alpha \pm \frac{v}{c} \Delta t \sqrt{\cos ^{2} \alpha-\left(1-\frac{c^{2}}{v^{2}}\right)}
$$

For the special case of $\alpha=0 \ldots, \ldots \pi$ we can get similar result for the Doppler shift obtained from the special relativity theory. For $\alpha=\pi / 3$ one obtains $v=c$ and $\Delta t^{\prime}=\Delta t$ which correspond to photons.

For the special case of $\alpha=\pi / 2$ one obtain the contraction time relation, which is normally obtained from the special relativity, but here the interpretation is a little bit different. In our case, we do not use the Lorentz transformation since we are not talking about passing from one reference frame to another, rather one consider that the second clock is also at the object but measuring a different event (the time of awareness).

Our result is based on Fermat' principle where the distance $A B$ ' is a minimum. The correct formulation involves the minimal total optical length for the wave propagation if we considerer the phase invariance $\omega^{\prime} \Delta t^{\prime}=\omega \Delta t=$ const. It seems they produce a full awareness system of communication.

In this paper we will use the contraction result which is obtained when $\alpha=\pi / 2$, equation (2), that is $\Delta t^{\prime}=\Delta t \sqrt{1-\frac{v^{2}}{c^{2}}}$ to estimate one relativistic effect on clocks aboard GPS satellite. This correction due to its orbital speed is similar 
to the special contraction relationship but here we are not using the special relativity theory.

In this last theory are considered two inertial Cartesian frames of reference, each of them having a clock fixed at the origin and one of them moving relative with a constant velocity $\mathrm{v}<\mathrm{c}$ along a common axis (i e $\mathrm{x}$-axis). The clocks are calibrated so that they point to the same time $t=t^{\prime}=0$ when the frames coincide. Thus by direct algebra, can be obtained the well-known time dilation formula $t=t^{\prime} / \sqrt{1-\frac{v^{2}}{c^{2}}}$ of special relativity.

One of the major influences upon its rate of timekeeping, is a correction for its orbital speed (velocity effect).

As observed by an earthbound receiver, the transmitting clock is subject to time dilation due to its orbital speed. A clock aboard a spaceship traveling at speed $v$ runs slow (compared to a stationary clock) by a factor which can be obtained when $\alpha=\pi / 2$ in equation (2)

$$
\frac{\Delta t^{\prime}}{\Delta t}=\sqrt{1-\frac{v^{2}}{c^{2}}} \approx 1-\frac{v^{2}}{2 c^{2}}
$$

Provided $v \ll c$ as would be the case for a satellite.

Thus when one second of proper times elapses, the moving clock loses $\frac{v^{2}}{2 c^{2}}=K / E_{0}$ seconds, where $K$ and $E_{0}$ are the kinetic and the rest energies of the clock, respectively. Thus we have

$$
\frac{\Delta t}{\tau}=\frac{v^{2}}{2 c^{2}}=K / E_{0}
$$

Where $\Delta t$ is the time lost by the orbiting clock when a interval time $\tau$ elapses on the surface-bound clock. That is a special relativistic correction for its orbital speed (velocity effect).

\section{GENERAL RELATIVISTIC EFFECT ON CLOCK ABOARD GPS SATELLITES}

Considerer a clock aboard a satellite orbiting the Earth such a global positioning system, GPS transmitter. There is another major relativistic influences upon its rate of timekeeping, a general relativistic correction for its orbital altitude (gravitational effect).

This effects can be treated at an introductory level, making for an appealing application of relativity to every life.

A clock at the higher gravitational potential of orbit runs faster than a surface clock. The gravitational potential energy of a body of mass $m$ in a Earth's gravity is $U=m V$ (see equation (11a) of Appendix A). $h_{00} / 2=-V=+\frac{G M_{E}}{r}$ where $V=-\frac{G M_{E}}{r}$ is Earth's gravitational potential (at distance $r$ from the center of the earth of mass $M_{E}$ ). In the case of a photon, we replace m by $E / c^{2}$, where $E=h f$ is the photon energy, where $\mathrm{h}$ is the Planck's constant and $\mathrm{f}$ is the frequency. If the photon travels downward in Earth's gravitational field, it therefore loses potential energy of $\left(h f / c^{2}\right) \Delta V$ and gains an equal amount of kinetic energy $h \Delta f$. We thereby deduce that the falling photon is gravitationally blue-shifted by $\Delta f=f \Delta V / c^{2}$.

This expression can also be straightforwardly deduced using the equivalence principle. If the clock's ticking is synchronized to a light wave, the orbiting clock will be observed at Earth's surface to be ticking faster due to this gravitational frequency shift.

Therefore when one second of earth time elapses, the clock at high altitude gains $\Delta V / c^{2}=\Delta U / E_{0}$ seconds, where $U$ is the gravitational potential energy of the clock.

Thus we have

$$
\frac{\Delta t}{\tau}=\frac{\Delta V}{c^{2}}=-\frac{\Delta U}{c^{2}}
$$

The sum of the two effects can be compactly expressed as

$$
\frac{\Delta t}{\tau}=\frac{K-U}{E_{0}}
$$

Where $\Delta t$ is the time lost by the orbiting clock when a time interval $\tau$ elapses on the surface-bound clock. Here $K-U$ is the Lagrangian of the orbiting clock where the reference level for the gravitational potential energy is chosen to lie at Earth's surface. 


\section{EARTHQUAKE PHENOMENA: BASIC THEORY AND DETERMINATION OF EARTHQUAKE MAGNITUDES}

Basic theory: There are two classes of seismic waves: body waves and surface waves. Body waves travel through the interior of the Earth. Two types of body waves are recognized: $P$ waves and $S$ waves. $P$ waves are the fastest seismic waves, and consequently, the first to arrive at any given location. Because of this fact, they were initially referred to as the primary waves of an earthquake. "Primary" was later shortened simply to "P". Travelling at a speed typically around $60 \%$ that of $\mathrm{P}$ waves, $\mathrm{S}$ waves always arrive at a location after them - the "S" stands for secondary. As with sound, the speed at which seismic waves travel depends upon the properties of the matter through which they propagate. In general, the less dense the matter, the slower the waves. P waves, also known as push waves, result from forward movement. Torsion waves, often called $\mathrm{S}$ waves, represent the spiraling motion of particles twisting between inner structures. $\mathrm{P}$ waves are usually the first to be recorded on a seismogram because they travel the fastest. S waves usually have more height, or amplitude, than $\mathrm{P}$ waves. The amplitude of the waves can help to reveal information about the magnitude of an earthquake and the circular polarization can reveal the origin of earthquake.

There are many geophysical precursors of earthquakes; the most important geophysical precursors are: the velocity of the P-wave changes, ground uplift and tilt, radon emissions increase, electrical resistivity of rocks decrease, and underground water level fluctuates. We are interested in the first geophysical precursor, which may be related with our proposal. The change in the velocity of the $\mathrm{P}$-wave is found by measuring the change in the ratio of the P-wave velocity to the S- wave velocity $V_{p} / V_{s}$. The $V_{p} / V_{s}$ ratio is obtained from an analysis of the travel times of P- and S- waves [1]. Denoting the arrival times of $\mathrm{P}$ - and S-waves by $t_{p}$ and $t_{s}$ respectively, the S-P time versus $t_{p}$ relation can be expressed by a straight line on the $\left(t_{p}-t_{s}\right)-t_{p}$ plane. The inclination $(k)$ of the line is given as: $k=-\left(t_{p}-t_{s}\right) / t_{p}=\Delta t / t_{p}$. If the propagation path for both waves is assumed to be identical, we obtain: $V_{p} t_{p}=V_{s} T_{s}$ so that we have: $k=V_{p} / \mathrm{V}_{\mathrm{s}}-1$. Therefore, it is seen that the $V_{p} / \mathrm{V}_{\mathrm{s}}$ ratio is obtained from $k$ calculated on the basis of travel-time analysis.
Starting from such concepts our aim will be to find a correlation and to support the following empirical relation of Rikitake: $\log \Delta t=0.75 M-4.27$ where $\Delta t$ (in the sense of our comprehension is $\Delta t^{\prime}$ ), is the time interval measured in years between the detection of anomalous land-deformation and an earthquake occurrence. $\mathrm{M}$ is the magnitude of the quake concerned.

Here we assume that the magnitude of an earthquake depends on our information on time starting from the instant when irregularities of the dilatation function take place to the instant of the earthquake occurrence. On the other hand, we know, according to Bullen, [2], the damping effect may be represented by the presence of a factor $\rho^{-k D}$, where $\mathrm{D}$ is the distance travelled by a wave and $k$ is the wave number of the $\mathrm{P}$-wave which is the order of $10^{-4} \mathrm{~km}^{-1}$. The interval in which the P-waves travel to $k^{-1}$ is $k^{-1} / V_{p}=k^{-1} /((\lambda+2 \varepsilon) / \rho)^{1 / 2}$.

Where $V_{P}$ is the velocity of P-waves, $\rho$ the mass density and $\lambda, \varepsilon$ elastic parameters.

The magnitude of an earthquake will be supposed also to depend on $\tau$ and given by $M=\sigma \ln \frac{\Delta t^{\prime}}{\tau}=\sigma \ln \frac{\Delta t \rho^{-\bar{\chi}(x)}}{\tau}$.

Here $\sigma$ is a certain constant so $\ln \Delta t^{\prime} \approx \sigma^{-1} M-10$. If we take $V p=7 \mathrm{~km} / \mathrm{s}$ and $\tau \approx 1.43 \times 10^{3}$, which is a good approximation to $\ln \Delta t=0.75 M-4.27$ if $\sigma^{-1}=1.7$.

\section{Determination of earthquake magnitudes}

When considering the problem of electromagnetic emissions correlated with earthquakes, one is often faced with an overwhelming problem of complexity, because the mechanism of wave generation is not entirely understood. Several observations of Very Low Frequency (VLF) emissions apparently associated with earthquakes, are recorded independently at ground-based stations and on satellites.

Recently, Hara [3-4], developed a new method to determine earthquake magnitudes using the following formula: $M=\alpha \log A_{e q}+\beta \log L+\gamma \log \Delta t+\delta$, where $\mathrm{M}$ is an earthquake magnitude, $A_{e q}$ is the maximum displacement during high-frequency energy radiation from the arrival time of a $\mathrm{P}$-wave, $\mathrm{L}$ is the epicentral distance, $\Delta t$ is duration of high-frequency energy radiation. The duration of highfrequency energy radiation can be estimated by band-pass filtering of first arriving P-waves, $\alpha, \beta, \gamma, \delta$ are $0.79,0.83$, 0.69 , and 6.47 , respectively (the units of $A_{e q}, L, \Delta t$ are m, $\mathrm{km}$, and s, respectively). 
He applied this method to the November 14, 2007 Antofagasta, Chile Earthquake. Data of measurements of high-frequency energy radiation show that The estimated duration is $81.6 \mathrm{sec}$. The estimated magnitude using the above formula is 7.91 .

Also, he applied this method to the February 27, 2010, Chile Earthquake (the origin time: 06:34:14 UTC; the location $35.846^{\circ} \mathrm{S}, 72.719^{\circ} \mathrm{W}$ after USGS). Data of measurements of high-frequency energy radiation show that the estimated duration is $138.6 \mathrm{sec}$. The estimated magnitude using the above formula is 8.57 .

Although this estimate is smaller than 8.8 from the Global CMT and USGS WPhase MT, it is consistent with them considering its uncertainly (around 0.2 in magnitude unit).

\section{On the earthquake as cosmological manifestation and method of predicting earthquakes}

Our main goal is to define the connection between the Rikitake's theory (seismic P-waves), and the Hara's relation (high-frequency energy radiation from the arrival time of a P-wave). This connection is that the factor $\Delta t^{\prime}=\Delta t / \xi(x)$ is equal to equation (3). The meaning of time may be considered in two ways: (a) the time based on the reading of clocks which suffer dilatation due to chiral fields [5-6], and (b) the time as it is accepted as in special relativity. In order to obtain reproduction of the fixed unit of time relating to the world which would nor suffer dilatation (chiral factor $\mathrm{T}=0$ ), we can introduce a time space function $\xi(x)$ which transforms an interval of proper time $\Delta t$ in our information on time according to $\Delta t^{\prime}=\Delta t / \xi(x)$ where we have $\xi(x)=1+\bar{\chi}$, $\bar{\chi}=\chi+\delta \chi(x)$ and $\delta \chi(x)$ take into account the occurrence of earthquake phenomena. Here, we propose two hypotheses: a) the chiral potential (T parameter) of a chiral Weyl's theory [5-6], is unambiguously related to the cosmological dilatation function $\xi(x)$ and the radiation eigenwaves are circular polarized waves with the same polarization of rotational seismic waves; b) the difference in time $\Delta t$ in the chiral Weyl's theory is the same as the time variable of time involved in the Hara's formula, $\left(M=\alpha \log A_{e q}+\beta \log L+\gamma \log \Delta t+\delta\right)$, and the empirical relationship of Rikitake $(\ln \Delta t=0.75 M-4.27)$.

Then, if the process leading to the occurrence of the earthquake influences changes of our information on time, then possibility of its prediction can be suggested.
If we consider a large plate of Earth's crust, for example from Antofagasta, point $P_{1}$ and Arica, point $P_{2}$, the dilatation function $\xi$ obtained from the chiral Einstein's theory gives a difference of the cosmological forces between $P_{1}$ and $P_{2}$.

If $\xi$ or $\delta \chi(x)$ is properly correlated with the $A_{e q}$ factor obtained from the solution of the $\mathrm{P}$-waves equation and $\mathrm{M}$, then we are in a position to propose an original prediction system as a early detection system for earthquakes.

Considering two atomic clocks $C_{1}$ and $C_{2}$ at points $P_{1}$ and $P_{2} . C_{1}$ and $C_{2}$ starting at the same time and at any subsequent instant, as their distances of the Earth's center are approximately equal, they give the same information on time $T_{1}{ }^{\prime}=T_{2}{ }^{\prime}$. However if the at point $P_{1}$ the adhesion between adjacent plates is smaller than at point $P_{2}$ then the difference in forces due to the relative dilatation $\xi(x)$, give a time difference of atomic clocks, that is if $\Delta T_{12}{ }^{\prime}=T_{12}{ }^{\prime}-T_{12}{ }^{\prime} \neq 0$ appears, then an earthquake can be expected either in the neighbourhood of $P_{1}$ or $P_{2}$.

This uncertainty can be removed with the aid of a third clock $C_{3}$ at any point $P_{3}$.

In general, if a certain region could be covered by $\mathrm{n}$ clocks at any $\mathrm{Ci}$ we should form $\sum_{\mathrm{k}} \Delta T_{i k}$ ' for $\mathrm{Ci}$ where $\sum_{\mathrm{k}} \Delta T_{i k}{ }^{\prime} \rightarrow \max$ we should have a point at which an earthquake can be expected. Our proposed work is find the correct relationship between $\delta \chi(x)$ or $\bar{\chi}(x)$ and $A_{e q}$, is the maximum displacement during high-frequency energy radiation from the arrival time of a $\mathrm{P}$-wave.

\section{APPENDIX A}

\section{A.0 Simple approach to general relativity}

With this material we are going to study Astrophysical Phenomena under the influence of gravity, e.g. precession of perihelion of planets, light deflection, particle motion in the vicinity of black hole, cosmology and other applications as in our case the relativistic effect on GPS systems due to the orbital altitude on clock aboard GPS satellites [7-9]. General Relativity is based on two principles:

(1) Principle of Equivalence and (2) Einstein equations The former tells us that the effect of gravity can be replaced by the effect of a curved space-time. The latter tells us how the matter distribution affects the space-time metric. 


\section{A.1 Principle of equivalence}

The origin of the equivalence principle goes back to the experiment of Galileo. The analysis of Galileo's experiment proceeds as follows. The force acting on a mass $m_{g}$ in a gravitational field $g$ is

$$
F=m_{g} g\left(g=\frac{G M}{R^{2}}\right)
$$

Then according to Newton's second law of motion the acceleration $a$ of this mass is given by $F=m_{i} a, m_{i}$ is the inertial mass and $m_{g}$ is the gravitational mass. Eliminate $F$ from above two equation gives:

$$
a=\left(\frac{m_{g}}{m_{i}}\right) g
$$

The first careful experiments specifically designed to test the equality of inertial and gravitational mass are due to Newton.

\section{A.2 Space-time metric}

In Newtonian mechanics, the motion of a particle can be expressed as 4 space-time variables $(t, \vec{x})$ and time $t$ is absolute. In special relativity time is no longer absolute.

In fact, the particle motion should be a function of $x^{\mu}(\tau)$ where $x^{\mu}=(c t, \vec{x})$ and $\tau$ is the proper time.

In Minkowski space (4-D) there is an invariant interval, namely,

$$
\begin{gathered}
d \tau^{2}=d t^{2}-\frac{1}{c^{2}}\left(d x^{2}+d y^{2}+d z^{2}\right) \\
=d t^{2}-\frac{1}{c^{2}}\left[d r^{2}+r^{2}\left(d \theta^{2}+\sin ^{2} \theta d \phi^{2}\right)\right]
\end{gathered}
$$

Since the choice of coordinate is arbitrary, we can express the invariant interval in any arbitrary coordinate system. Here the matrix $g_{i j}$ is called "Metric Tensor". $\eta^{\mu v}$ is also called Minkowski metric.

(In Special Relativity (e.g. book of Weinberg), $x^{\mu}=(c t, \vec{x})$,

$$
x^{\mu}=(c t,-\vec{x}), \eta^{\mu v}=\left(\begin{array}{cccc}
-1 & & & \\
& 1 & & \\
& & 1 & \\
& & & 1
\end{array}\right)=\eta_{\mu v}
$$

The metric tensor in 4-D

$$
g_{\mu v}=\eta_{\alpha \beta} \frac{\partial \xi^{\alpha}}{\partial x^{\mu}} \frac{\partial \xi^{\beta}}{\partial x^{v}}
$$

If $\left(x^{0}, x^{1}, x^{2}, x^{3}\right)=(-c t, x)$

$$
g_{\mu v}=\left(\begin{array}{rrrr}
-1 & 0 & 0 & 0 \\
0 & 1 & 0 & 0 \\
0 & 0 & 1 & 0 \\
0 & 0 & 0 & 1
\end{array}\right)=\eta_{\mu v}
$$

So far, the gravity is not included. To denote the coordinate system (observer) without gravity, the metric tensor is $\eta_{\alpha \beta}$. Otherwise $g_{\mu \nu}$ is more general.

\section{A.3 Equation of motion (particles with $\boldsymbol{m} \neq 0$ ) under gravity.}

If a particle is freely falling in a gravitational field, its equation of motion should be

$$
\frac{d^{2} \xi^{\alpha}}{d \tau^{2}}=0
$$

where $\xi^{\alpha}$ is the coordinate in the free-falling frame and $d \tau$ is the proper time which satisfies $c^{2} d \tau^{2}=-\eta_{\alpha \beta} d \xi^{\alpha} d \xi^{\beta}$.

For an arbitrary observer who is not free falling, his coordinate is $x^{\mu}$. We can express $\xi^{\alpha}$ in terms of $x^{\mu}$ in equation (1)

$$
0=\frac{d}{d \tau}\left[\frac{\partial \xi^{\alpha}}{\partial x^{\mu}} \frac{d x^{\mu}}{d \tau}\right]=\frac{\partial \xi^{\alpha}}{\partial x^{\mu}} \frac{d^{2} x^{\mu}}{d \tau^{2}}+\frac{\partial^{2} \xi^{\alpha}}{\partial x^{v} \partial x^{\mu}} \frac{d x^{\mu}}{d \tau} \frac{d x^{v}}{d \tau}
$$

Multiply $\frac{\partial x^{\lambda}}{\partial \xi^{\alpha}}$ to the above equation, we obtain

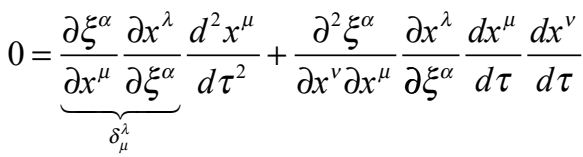

$$
\begin{aligned}
& =\delta_{\mu}^{\lambda} \frac{d^{2} x^{\mu}}{d \tau^{2}}+\frac{\partial^{2} \xi^{\alpha}}{\partial x^{v} \partial x^{\mu}} \frac{\partial x^{\lambda}}{\partial \xi^{\alpha}} \frac{d x^{\mu}}{d \tau} \frac{d x^{v}}{d \tau} \\
& =\frac{d^{2} x^{\lambda}}{d \tau^{2}}+\Gamma_{\mu \nu}^{\lambda} \frac{d x^{\mu}}{d \tau} \frac{d x^{v}}{d \tau}
\end{aligned}
$$

where

$$
\Gamma_{\mu v}^{\lambda} \equiv \frac{\partial x^{\lambda}}{\partial \xi^{\alpha}} \frac{\partial^{2} \xi^{\alpha}}{\partial x^{v} \partial x^{\mu}}=\Gamma_{v \mu}^{\lambda}
$$


The quantity $\Gamma_{\mu v}^{\lambda}$ is called the Christoffel symbol or the affine connection.

Similarly,

$$
d s^{2}=c^{2} d \tau^{2}=-g_{\mu v} d x^{\mu} d x^{v}
$$

Equation (2a) is the equation of motion of particle in an arbitrary frame. In relativity, the equation of motion is also called the geodesic equation.

For $m=0$ (e.g. photon), the equation of motion is the same as that of equation (2a). However, the independent variable cannot be the proper time because for massless particles $d \tau^{2}=0$.

In this case we choose an arbitrary parameter along the world line, say $\rho$, whose physical meaning is not important. Equation (2a) becomes

$$
\frac{d^{2} x^{\lambda}}{d \rho^{2}}+\Gamma_{\mu \nu}^{\lambda} \frac{d x^{\mu}}{d \rho} \frac{d x^{\nu}}{d \rho}=0
$$

and

$$
d \tau^{2}=0 \Rightarrow 0=g_{\mu v} \frac{d x^{\mu}}{d \rho} \frac{d x^{v}}{d \rho}
$$

In general

$$
g_{\mu v}=\frac{\partial \xi^{\alpha}}{\partial x^{\mu}} \frac{\partial \xi_{\alpha}}{\partial x^{v}}=\eta_{\alpha \beta} \frac{\partial \xi^{\alpha}}{\partial x^{\mu}} \frac{\partial \xi^{\beta}}{\partial x^{v}}
$$

Actually, the real meaning of $\tau$ and/or $\rho$ is not important. After we solve equations (4a)/(5a), we get $x^{\mu}(\tau) / x^{\mu}(\rho)$. We can use equations (3a)/(4a) to eliminate $\tau / \rho$ to get $x^{i}(t)$.

\section{A.4 The relation between $g^{\mu v}$ and $\Gamma_{\mu v}^{\alpha}$}

Let's differentiate $g_{\mu \nu}$ with respect to $x^{\lambda}$

$$
\begin{aligned}
& \frac{\partial}{\partial x^{\lambda}} g_{\mu \nu}=\frac{\partial^{2} \xi^{\alpha}}{\partial x^{\lambda} \partial x^{\mu}} \frac{\partial \xi^{\beta}}{\partial x^{\nu}} \eta_{\alpha \beta}+\frac{\partial \xi^{\alpha}}{\partial x^{\mu}} \frac{\partial^{2} \xi^{\beta}}{\partial x^{\lambda} \partial x^{\nu}} \eta_{\alpha \beta} \\
& =\Gamma_{\lambda \mu}^{\rho} \underbrace{\frac{\partial \xi^{\alpha}}{\partial x^{\rho}} \frac{\partial \xi^{\beta}}{\partial x^{v}} \eta_{\alpha \beta}}_{g_{\rho v}}+\Gamma_{\lambda v}^{\rho} \underbrace{\frac{\partial \xi^{\alpha}}{\partial x^{\mu}} \frac{\partial \xi^{\beta}}{\partial x^{\rho}} \eta_{\alpha \beta}}_{g_{\rho \mu}}
\end{aligned}
$$

we have used the definition of $\Gamma_{\mu \nu}^{\alpha}$ and

$$
\begin{aligned}
\left(\frac{\partial x^{\lambda}}{\partial \xi^{\alpha}}\right)^{-1} & =\frac{\partial \xi^{\alpha}}{\partial x^{\lambda}} \\
& \Rightarrow \frac{\partial}{\partial x^{\lambda}} g_{\mu v}=\Gamma_{\lambda \mu}^{\rho} g_{\rho \nu}+\Gamma_{\lambda v}^{\rho} g_{\rho \mu}
\end{aligned}
$$

From equation (5a), we can show

$$
\begin{gathered}
\frac{\partial g_{\mu v}}{\partial x^{\lambda}}+\frac{\partial g_{\lambda v}}{\partial x^{\mu}}-\frac{\partial g_{\mu \lambda}}{\partial x^{v}}= \\
=\left(g_{\rho v} \Gamma_{\lambda \mu}^{\rho}+\Gamma_{\lambda v}^{\rho} g_{\rho \mu}\right)+\left(g_{\rho v} \Gamma_{\mu \lambda}^{\rho}+\Gamma_{\mu \nu}^{\rho} g_{\rho \lambda}\right)+ \\
-\left(g_{\rho \lambda} \Gamma_{v \mu}^{\rho}+\Gamma_{\nu \lambda}^{\rho} g_{\rho \mu}\right) \\
=2 g_{\rho v} \Gamma_{\mu \lambda}^{\rho}
\end{gathered}
$$

We can also see that

$$
g^{v \sigma} g_{\rho v}=\delta_{\rho}^{\sigma}
$$

$\left(g_{\alpha \mu} G^{\mu \beta}=\delta_{\alpha}^{\beta} g\right.$ where $G^{\mu \beta}$ and $g$ are the cofactor and determinant of $g_{\alpha \mu}$. Let us write $g^{\alpha \beta}=\frac{G^{\alpha \beta}}{g}$-associate
tensor of $g_{\alpha \beta}$ ). Take $g^{v \sigma}$ multiply equation (6a), we obtain

$$
\Gamma_{\mu \lambda}^{\sigma}=\frac{1}{2} g^{v \sigma}\left(\frac{\partial g_{\mu v}}{\partial x^{\lambda}}+\frac{\partial g_{\lambda v}}{\partial x^{\mu}}-\frac{\partial g_{\mu \lambda}}{\partial x^{v}}\right)
$$

(The metric tensor $g_{\mu \nu}$ is known after solving the Einstein equations)

\section{A.5 Newtonian limit}

For a slowly moving particle $\frac{d x^{i}}{d \tau}<<c$, the equation of
motion becomes

$$
\frac{d^{2} x^{\mu}}{d \tau^{2}}+\Gamma_{00}^{\mu}\left(\frac{d t}{d \tau}\right)^{2}=0
$$


For static gravitational field, any time derivative of $g_{\mu v}$ is zero. Similarly, we can show

$$
\begin{aligned}
& \Gamma_{00}^{\mu}=\frac{1}{2} g^{\mu v}(\underbrace{\frac{\partial g_{v 0}}{\partial x^{0}}}_{=0}+\underbrace{\frac{\partial g_{0 v}}{\partial x^{0}}}_{=0}-\frac{\partial g_{00}}{\partial x^{v}}) \\
& =-\frac{1}{2} g^{\mu \nu} \frac{\partial g_{00}}{\partial x^{v}}
\end{aligned}
$$

Since we have assumed the field is weak,

$$
\begin{gathered}
g_{\alpha \beta} \approx \eta_{\alpha \beta}+h_{\alpha \beta},\left[h_{\alpha \beta}\right]<<1 \\
\Rightarrow \Gamma_{00}^{\alpha}=-\frac{1}{2} g^{\alpha \beta} \frac{\partial g_{00}}{\partial x^{\beta}}=-\frac{1}{2} \eta^{\alpha \beta} \frac{\partial h_{00}}{\partial x^{\beta}}
\end{gathered}
$$

Substitute this into equation of motion (*) and set $\mu=0$

$$
0=c^{2} \frac{d t^{2}}{d \tau^{2}}-\frac{1}{2} \eta^{0 \beta} \frac{\partial h_{00}}{\partial x^{\beta}}=c^{2} \frac{d^{2} t}{d \tau^{2}}
$$

Let's consider $\mu=1,2,3$, we have

$$
\frac{d^{2} x^{i}}{d \tau^{2}}+\Gamma_{00}^{i}\left(\frac{d t}{d \tau}\right)^{2}=0
$$

where

$$
\begin{aligned}
\Gamma_{00}^{i}=-\frac{1}{2} \eta^{i \beta} \frac{\partial h_{00}}{\partial x^{\chi}} & =-\frac{1}{2} \eta^{i i} \frac{\partial h_{00}}{\partial x^{i}}=-\frac{1}{2} \nabla_{i} h_{00} \\
& \Rightarrow \frac{d^{2} x^{i}}{d \tau^{2}}=\frac{1}{2}\left(\frac{d t}{d \tau}\right)^{2} \nabla_{i} h_{00}
\end{aligned}
$$

The solution of equation $\left(^{*}\right)$ is simple, it is

$$
\frac{d t}{d \tau}=\frac{1}{\sqrt{-g_{00}}}=\text { const }
$$

(The metric equation $-c^{2} d \tau^{2}=g_{\mu \nu} d x^{\mu} d x^{v} \Rightarrow\left(\frac{d \tau}{d t}\right)^{2}=-g_{00}$ )

Substitute this into equation (9a)

$$
\frac{d^{2} x^{i}}{d t^{2}}=\frac{1}{2} \nabla_{i} h_{00}
$$

Comparing this expression with Newtonian force equation, we conclude that

$$
h_{00}=-2 V=+2 \frac{G M}{r}
$$

Since $g_{00} \approx-1+\mathrm{h}_{00}$, equation (10a) implies that $r=$ const.

$$
r=\text { const. }
$$

It means that particle under the static gravitational force will do a circular motion in spherical symmetric case. (This assumption is hidden in static field assumption otherwise $g^{\mu v}$ is not constant seen by the particle).

\section{A.6 Time dilation and red shift}

a) Time dilation (expansion)

In a free fall frame, the proper time interval is given by

$$
\begin{gathered}
d \tau=\left(-\eta_{\alpha \beta} \frac{d \xi^{\alpha}}{c} \frac{d \xi^{\beta}}{c}\right)^{\frac{1}{2}} \\
=(-\underbrace{\eta_{\alpha \beta} \frac{\partial \xi^{\alpha}}{\partial x^{\mu}} \frac{\partial \xi^{\beta}}{\partial x^{v}} \frac{d x^{\mu}}{c} \frac{d x^{v}}{c}}_{g_{\mu v}})^{\frac{1}{2}}
\end{gathered}
$$

(in an arbitrary frame $x^{\mu}$ )

$$
\Rightarrow \frac{d t}{d \tau}=\left(-g_{\mu \nu} \frac{d x^{\mu}}{c d t} \frac{d x^{v}}{c d t}\right)^{-\frac{1}{2}} \text { (actually this is a }
$$

world metric equation)

In a rest frame $\frac{d x^{i}}{d t}=0$, we have

$$
\frac{d t}{d \tau}=\left(-g_{00}\right)^{\frac{1}{2}}
$$

In weak field case, $-g_{00} \approx 1+2 \varphi<1, \Rightarrow d t>d \tau$, Therefore, $\left(-g_{00}\right)^{-\frac{1}{2}}$ is called the expansion factor. This means that the gravitational field tends to make the time interval longer. 


\section{CONCLUSIONS}

Two of the most important relativistic effects (to order $1 / \mathrm{c}^{2}$ ), the Doppler red shift of second order, due to the motion of the satellite (special relativity) and the Einstein gravitational blue shift effect of the satellite clock frequency (equivalence principle of general relativity) were analysed, related with GPS systems.

Both of these effects were treated at a basic level without resorting to the full theory of special and general relativity. One of the effects can be treated with our proposed time contraction approach and the other using the Newton's theory as an approximation of the General Relativity. These approaches allow us to propose an outline for early prediction and detection of strong earthquake phenomena. The main goal is to define and determine the connection between the Rikitake's theory (seismic P-waves), and the Hara's relation (high-frequency energy radiation from the arrival time of a $\mathrm{P}$-wave). The proposed detection system gives information of the maximum displacement of an earthquake during highfrequency energy radiation arising from the arrival time of a P-wave at a specific location.

\section{REFERENCES}

[1] T. Rikitake. "Earthquake Prediction". Elsevier Scientific Publishing Company, Amsterdam. The Netherlands. 1976.

[2] K.E. Bullen and B.A. Bolt. "An introduction to the theory of seismology". Cambridge University Press. 1959.
[3] T. Hara. "Measurement of duration of highfrequency energy radiation and its application to determination of magnitudes of large shallow earthquakes". Earth Planets Space. Vol. 59, pp. 227-231. 2007.

[4] T. Hara. "Magnitude determination using duration of high frequency energy radiation and displacement amplitude: application to tsunami earthquakes". Earth Planets Space. Vol. 59, pp. 561-565. 2007.

[5] H. Torres-Silva. "Electrodinámica Quiral: Eslabón para la Unificación del Electromagnetismo y la Gravitación". Ingeniare. Revista chilena de ingeniería. Vol. $16 \mathrm{n}^{\circ}$ especial, pp. 6-23. 2008. URL: http://www. scielo.cl/scielo. php?script $=$ sci_arttext\&pid $=$ S0718$33052008000400002 \& \operatorname{lng}=$ es\&nrm $=$ iso $>$. doi: 10.4067/S0718-330520080004 00002.

[6] H. Torres-Silva. "Extended Einstein's Theory of Waves in the Presence of Space-Time Tensions". Ingeniare. Revista chilena de Ingeniería. Vol. 16 $\mathrm{n}^{\circ}$ especial, pp. 78-84. 2008. URL: http://www.scielo. cl/scielo.php? script $=$ sci_arttext $\&$ pid $=$ S0718330520080004 00012\&lng = es\&nrm = iso $>$. doi: 10.4067/S0718-33052008000400012.

[7] N. Ashby. "Relativity in the Global Positioning System". Living Reviews in Relativity". Vol. 6. 2003. URL: http://relativity.livingreviews.org/ Articles/lrr-2003-1/

[8] N. Ashby. "Relativity and the Global Positioning System”. Phys. Today. Vol. 55, pp. 41-47. May, 2002.

[9] A. Ashtekar. "100 Years of Relativity. SpaceTime Structure: Einstein and Beyond". World Scientific Publishing. 2006. 